\title{
Dual-comb coherent Raman spectroscopy with lasers of 1-GHz pulse repetition frequency
}

\author{
Kathrin J. Mohler, ${ }^{1,2}$ Bernhard J. Bohn, ${ }^{1,2}$ Ming Yan, ${ }^{1,2}$ Gwénaëlle Mélen, ${ }^{2}$ \\ Theodor W. Hänsch, ${ }^{1,2}$ and Nathalie Picqué ${ }^{1,2, *}$ \\ ${ }^{1}$ Ludwig-Maximilians-Universität München, Fakultät für Physik, Schellingstrasse 4/III, 80799 München, Germany \\ ${ }^{2}$ Max-Planck-Institut für Quantenoptik, Hans-Kopfermann-Strasse 1, 85748 Garching, Germany \\ ${ }^{*}$ Corresponding author: nathalie.picque@mpq.mpg.de
}

Received 10 November 2016; revised 9 December 2016; accepted 10 December 2016; posted 15 December 2016 (Doc. ID 279149); published 11 January 2017

\begin{abstract}
We extend the technique of multiplex coherent Raman spectroscopy with two femtosecond mode-locked lasers to oscillators of a pulse repetition frequency of $1 \mathrm{GHz}$. We demonstrate a spectra of liquids, which span $1100 \mathrm{~cm}^{-1}$ of Raman shifts. At a resolution of $6 \mathrm{~cm}^{-1}$, their measurement time may be as short as $5 \mu$ s for a refresh rate of $2 \mathrm{kHz}$. The waiting period between acquisitions is improved 10-fold compared to previous experiments with two lasers of 100-MHz repetition frequencies. () 2017 Optical Society of America
\end{abstract}

OCIS codes: (300.6300) Spectroscopy, Fourier transforms; (140.4050) Mode-locked lasers; (300.6450) Spectroscopy, Raman.

https://doi.org/10.1364/OL.42.000318

Laser frequency combs enable new approaches to molecular spectroscopy and sensing [1-12]. One of these approaches [7] harnesses coherent nonlinear Raman effects in condensed matter with a pump-probe scheme involving two femtosecond combs of slightly different pulse repetition frequencies. Dualcomb coherent anti-Stokes Raman spectroscopy (CARS) was demonstrated with spectra spanning around $1000 \mathrm{~cm}^{-1}$ and measured on a $\mu s-m s$ time scale with a resolution limited by the intrinsic width of the vibrational molecular bands.

Ref. [7] details the principle of dual-comb coherent Raman spectroscopy. In summary, in the time domain (Fig. 1), the pulses of one comb excite, with a periodicity of $1 /\left(f_{\text {rep }}+\delta f_{\text {rep }}\right)$, low-lying vibrational levels in a Raman two-photon process. The refractive index of the sample is modulated at the vibrational period with a ring-down time related to the coherence time of the transition. After each excitation, the pulse of a second comb of repetition frequency $f_{\text {rep }}$ probes the vibrational excitation at a linearly increasing time delay, which is automatically induced by the small difference in repetition frequencies $\delta f_{\text {rep }}$. The spectrum of the probe comb is alternately blue- and redshifted [13] and its intensity modulation, measured behind an edge spectral filter, directly reveals the frequencies of the excited molecular vibrations with a down-conversion factor equal to $\delta f_{\text {rep }} / f_{\text {rep }}$. A Fourier transform of such time-domain interference computes the Raman spectrum.

As in other dual-comb techniques, the time separation between two pulses of a pump-probe pair is periodically scanned by steps of $\delta f_{\text {rep }} / f_{\text {rep }}^{2}$ over a range from 0 to $1 / f_{\text {rep }}$. Therefore, for an optimal use of the experimental time, the observed decay time of the molecular sample (set by the vibrational coherence time or by the signal-to-noise ratio) should be similar to $1 / f_{\text {rep }}$. In the frequency-domain picture, the desired spectral resolution should be comparable to the comb line spacing. The ratio of the comb line spacing to the resolution gives the duty cycle of the acquisition sequence. As the coherence time of the molecular bands in the liquid phase is typically tens of ps, corresponding to line widths of several tens of $\mathrm{GHz}$ (several $\mathrm{cm}^{-1}$ ), frequency combs of large line spacing would benefit from dual-comb CARS. Moreover, the refresh rate $\delta f_{\text {rep }}$ of successive interferograms may be increased as its upper limit scales with the square of the repetition frequency $f_{\text {rep }}$. As detailed in [7], for a spectral span of $\Delta F$ (in $\mathrm{Hz}$ ) in the optical domain, $\delta f_{\text {rep }}$ must indeed be chosen less than or equal to $f_{\text {rep }}^{2} /(2 \Delta F)$ to fulfill the Nyquist

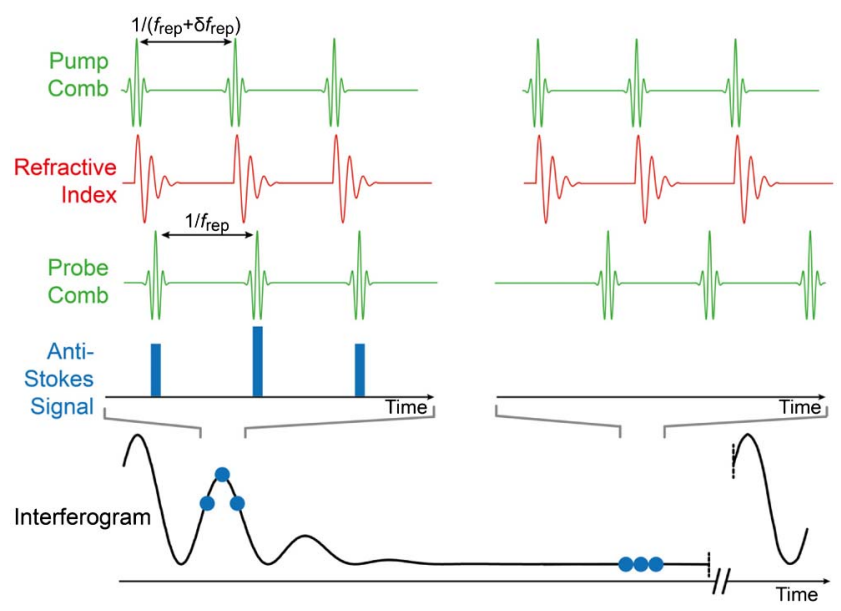

Fig. 1. Time-domain principle of dual-comb CARS. See text and [7] for details. 
criterion. However, coherent Raman four-wave mixing signals scale with the cube of the peak power of the laser pulses, making the use of high-repetition-frequency lasers challenging.

The first demonstrations [7] of dual-comb CARS have been performed with $100-\mathrm{MHz}$ oscillators of 20 -fs pulse duration and the duty cycle was limited to $3 \times 10^{-3}$. In this Letter, we investigate the potential of $1-\mathrm{GHz}$ oscillators for dual-comb CARS.

Figure 2 shows the experimental setup. Two identical titaniumsapphire femtosecond mode-locked lasers (Laser Quantum, Taccor-10s) are used. Their spectrum is centered around $795 \mathrm{~nm}$ $\left(12580 \mathrm{~cm}^{-1}\right)$ with a full width at half-maximum (FWHM) of $60 \mathrm{~nm}\left(880 \mathrm{~cm}^{-1}\right)$. At the output of the sealed oscillators, the pulses are chirped with a specified group delay dispersion of about $+450 \mathrm{fs}^{2}$. Their repetition frequency is around $1 \mathrm{GHz}$ and the difference in repetition frequencies $\delta f_{\text {rep }}$ is chosen between $500 \mathrm{~Hz}$ and $2000 \mathrm{~Hz}$. The repetition frequency is controlled by piezo-electric transducers, which adjust the laser cavity length. Low-bandwidth (about $10 \mathrm{kHz}$ ) active stabilization is achieved with a servo controller (Laser Quantum, TL-1000), which phase-locks the repetition frequency to an external reference provided by a radio-frequency synthesizer (Rohde \& Schwarz, SMA100A-B22). The root-mean-square fluctuations of the repetition frequency are $0.3 \mathrm{~Hz}$ over $5 \mathrm{~min}$ measured by a 12-digit counter with a gate time of $1 \mathrm{~s}$.

The two laser beams are linearly polarized with axes that are not perfectly orthogonal. They are combined on a pellicle beam splitter (Thorlabs, CM1-BP145B2). One output of the beam splitter is used for CARS, as detailed below. The second output measures the residual time-domain interference between the two femtosecond lasers on a fast photodiode (Thorlabs, PDA10A): the sequence of bursts at a period of $1 / \delta f_{\text {rep }}$ serves as a trigger signal for the acquisition of the dual-comb coherent Raman interferograms. On the CARS beam path, chirped mirrors (Layertec) compensate for the group delay dispersion $\left(-940 \mathrm{fs}^{2}\right.$ in total) in the entire setup (lasers, optical elements before the focal point, air). Spectral shearing interferometry is employed to characterize the ultrashort pulses by means of a SPIDER device (Venteon, Laser Quantum).

A combination of an optical long-wavelength-pass filter (Chroma, ET750lp, optical density: 5, cutoff: $750 \mathrm{~nm}-$ $13333 \mathrm{~cm}^{-1}$ ) before the sample, and a short-wavelength-pass filter (Omega Optical, 3RD750SP, transmission $<5 \%$ for wavelength $<750 \mathrm{~nm})$ after the sample separates the antiStokes radiation from the light of the titanium-sapphire lasers. The cutoff wavelengths can be slightly changed by tilting the interference filters. The beam is focused with an aspheric lens of a focal length of $8 \mathrm{~mm}$ (Newport, 5724) on the liquid sample contained in a $1-\mathrm{mm}$ thick quartz cuvette (Starna, Type 21). At the sample, for each laser the pulse duration is less than

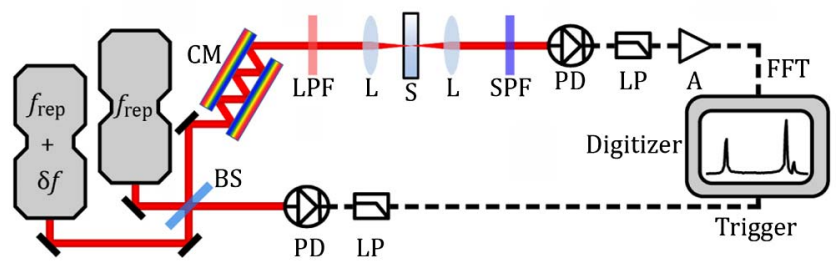

Fig. 2. Experimental setup for dual-comb CARS using two $1-\mathrm{GHz}$ lasers (see text for details). BS, beamsplitter; CM, chirped mirrors; LPF, long-pass filter; L, lens; S, sample; SPF, short-pass filter; PD, photodetector; LP, electronic long-pass filter; A, amplifier.
$20 \mathrm{fs}$ and the pulse energy is up to $0.5 \mathrm{~nJ}$. The anti-Stokes radiation emerging from the cuvette in the forward direction is collimated by a lens identical to the focusing one. It is optically filtered and focused onto a fast silicon photodiode (Newport, 1621). The interference signal is detected-together with a large background of other signals originating from unfiltered laser radiation-from nonlinear phenomena (including coherent Raman and non-resonant effects) generated at the sample by each laser and from residual intensity noise. The closeto-orthogonal polarizations of the two comb beams diminish the interferometric non-resonant background while retaining a significant part of the resonant contribution to the interference thanks to the depolarization of the sample. Moreover, the non-interferometric signals mostly occur at the repetition frequency of the lasers and their harmonics. Therefore, they can be electrically filtered out. After filtering (Mini Circuits, ZFHP-0R055-S+ and BLP-200+) and amplification (L-3 Narda-MITEQ, AU-1332), the time-domain interference signal is recorded by a data acquisition board (AlazarTech, ATS9360) with a rate of $10^{9}$ samples $\mathrm{s}^{-1}$.

The interferograms are processed by an automated custom MATLAB program. As in other Fourier transform CARS techniques [14-17], the interferometric non-resonant background is present only at short optical delays. It is, therefore, entirely suppressed by suitably positioning the numerical triangular apodization window. The length of the apodization window sets the resolution and the sixfold zero-filling of the interferogram interpolates the spectrum. Only the magnitude spectrum is computed. Phase correction is part of the improvements that need to be implemented in the future. When the two $1-\mathrm{GHz}$ lasers have similar pulse energies at the sample, each laser is alternately the pump and the probe, and the interferograms are symmetric about zero optical delays. For instance, the duty cycle, defined in [7] as the ratio $3.6 f_{\text {rep }} / \delta \nu$, where $\delta \nu$ is the optical resolution, is $2 \%$ for an apodized resolution of $6 \mathrm{~cm}^{-1}$. This is 10 -fold higher than with $100-\mathrm{MHz}$ systems. Here, however, due to a simplistic trigger of the data acquisition, only one side of the interferogram at about the zero optical delay is recorded and Fourier transformed, thus bringing the actual duty cycle to $1 \%$.

The Fourier transform reveals radio-frequency spectra. Their free spectral range varies from $21 \mathrm{MHz}\left(\delta f_{\text {rep }}=500 \mathrm{~Hz}\right.$, down-conversion factor: $\left.5 \times 10^{-7}\right)$ to $84 \mathrm{MHz}\left(\delta f_{\text {rep }}=\right.$ $2000 \mathrm{~Hz}$, down-conversion factor: $\left.2 \times 10^{-6}\right)$. The radiofrequency $\mathrm{x}$-scale is a posteriori converted to a scale of optical Raman-shifts by division by the down-conversion factor with the help of the experimentally measured repetition frequencies. The span of Raman shifts converted to wavenumbers is $300-1400 \mathrm{~cm}^{-1}$. It is limited on the low-wavenumber side by the optical filters and on the high-wavenumber side by the spectral bandwidth of the lasers. When spectra are averaged, the wavenumber axis of each individual spectrum is corrected by a multiplying factor prior to averaging. We determine the mean value of the experimental line positions in all the spectra and we multiply the $x$-scale of each spectrum by a factor that brings the positions to their mean value. This compensates for the residual fluctuations of the difference in repetition frequencies. Such fluctuations induce variations of a couple of $\mathrm{kHz}$ of the radiofrequency scale (up to $10 \mathrm{~cm}^{-1}$ in the wavenumber scale).

Figure 3 shows two experimental dual-comb CARS spectra of neat toluene measured within times on the microsecond 
scale. No averaging is performed. The apodized resolution is $6 \mathrm{~cm}^{-1}$. Therefore, the spectra comprise 183 spectral elements (defined as span divided by resolution). The duty cycle is $1 \%$ if one considers only single-sided interferograms. Five intense lines of toluene centered at $522 \mathrm{~cm}^{-1}, 788 \mathrm{~cm}^{-1}, 1001 \mathrm{~cm}^{-1}$, $1028 \mathrm{~cm}^{-1}$, and $1210 \mathrm{~cm}^{-1}$, respectively, are assigned [18] to the $\nu_{13}, \nu_{12}, \nu_{11}, \nu_{10}$, and $\nu_{8}$ bands, respectively. In Fig. 3(a), the difference in pulse repetition frequencies is $\delta f_{\text {rep }}=500 \mathrm{~Hz}$, which leads to a measurement time of $20 \mu \mathrm{s}$. The signalto-noise ratio for the strongest transition at $1001 \mathrm{~cm}^{-1}$ is 107 . The noise is taken as the standard deviation of the baseline in the wavenumber region around $1350 \mathrm{~cm}^{-1}$. Double-sided interferograms repeat at a refresh time of $1 / \delta f_{\text {rep }}=2 \mathrm{~ms}$. Every $2 \mathrm{~ms}$ it is possible to measure two spectra; each looks like that shown in Fig. 3(a) within $20 \mu$ s and with a time separation of a couple of $\mu$ s set by the apodization window. In Fig. 3(b), as $\delta f_{\text {rep }}=2000 \mathrm{~Hz}$, the measurement time is $5 \mu \mathrm{s}$, the signal-tonoise ratio is 50 , and the refresh time is $500 \mu$ s.

When we dilute toluene in 2-propanol, we find that the maxima of the toluene band profiles increase linearly with an increasing concentration. Moreover, we measure (Fig. 4) the dependence of the maximum of the benzene $\nu_{2}$ band at $992 \mathrm{~cm}^{-1}$ with the energy of the pump (resp. probe) pulses. When the energy of the pump (resp. probe) pulses at the sample is varied, the energy of the probe (resp. pump) pulses is kept constant to about $0.4 \mathrm{~nJ}$. We observe that the two curves almost overlap. A fit gives a slope of $1.04( \pm 0.02)$ for the energy dependence of the pump and $1.06( \pm 0.03)$ for that of the probe. The number in parentheses is the statistical uncertainty of the fit. Therefore, the line maxima depend linearly on the concentration of the sample and on the energy of both pump and probe pulses. With coherent Raman signals, a quadratic concentration dependence, as well as an overall cubic energy dependence, is expected. The observed linear behaviors imply that the detected signal results from the interference of the CARS electric field with another field [19,20]. The local oscillator in this additional interference mixing process is the probe beam whose pulses always coincide in time with the interferometric CARS signal at the detector. As the probe beam signal is concentration independent in such configuration, the detector signal is expected to be proportional to the concentration. The interferometric CARS signal scales with the square of pump

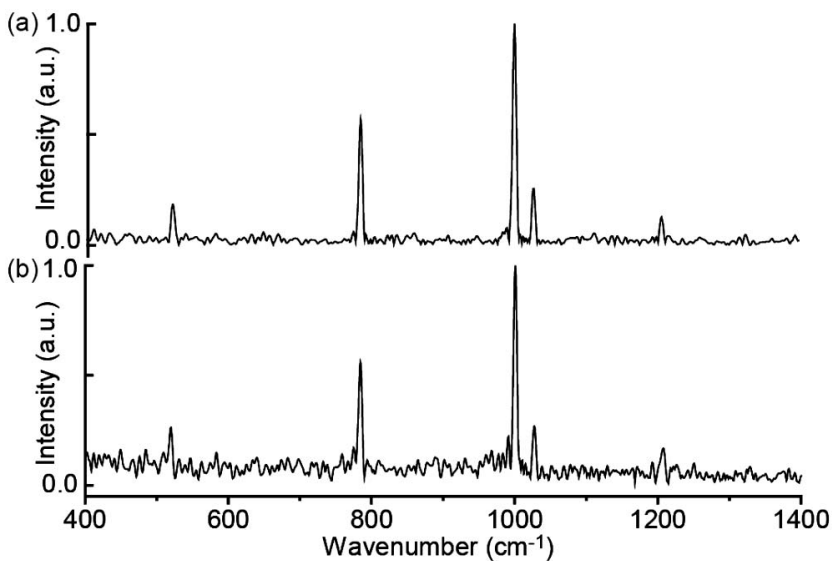

Fig. 3. Experimental spectra of toluene measured at $6 \mathrm{~cm}^{-1}$ resolution within (a) $20 \mu$ s and (b) $5 \mu$ s.

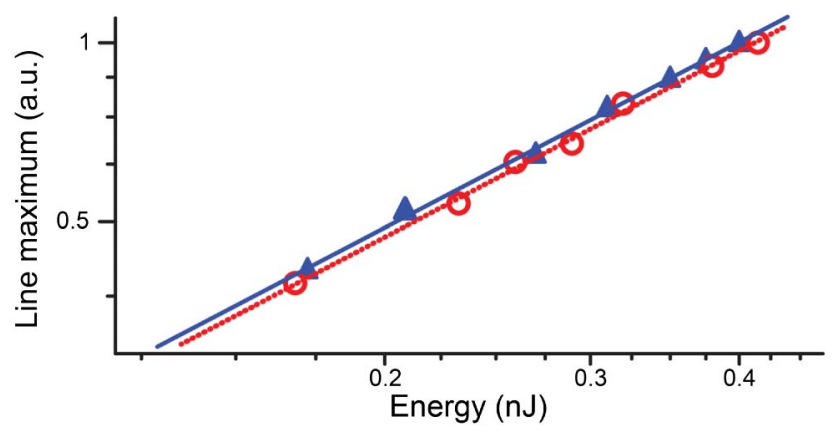

Fig. 4. Dependence of the maximum of the benzene $\nu_{2}$ line profile at $992 \mathrm{~cm}^{-1}$ with the energy of the pump (blue triangles) and probe (open red circles) pulses, on logarithmic $\mathrm{x}$ - and $\mathrm{y}$-scales.

pulse energy and with the probe pulse energy. The local oscillator signal scales with the probe pulse energy. Therefore, the detector signal, which results from the interferometric mixing between interferometric CARS and local oscillator signals, has a linear dependence with both the pump and probe pulse energies. In practice, the extent of spectral overlap at the detector between the mutually coherent probe and interferometric CARS signals is experimentally controlled by empirically adjusting the cutoff wavelengths of the optical filters placed before and after the sample. Such heterodyne detection of the weak CARS signal improves the signal-to-noise ratio and provides opportune linear concentration dependence.

Figure 5 presents the spectrum of a mixture of toluene, cyclohexane (band $\nu_{5}$ at $802 \mathrm{~cm}^{-1}$ [21]), and chloroform (band $\nu_{3}$ at $366 \mathrm{~cm}^{-1}$ and band $\nu_{2}$ at $678 \mathrm{~cm}^{-1}$ [22]). The difference in repetition frequencies is $\delta f_{\text {rep }}=600 \mathrm{~Hz}$. The apodized resolution is $6 \mathrm{~cm}^{-1}$ and 120 spectra are averaged. The measurement time is $2 \mathrm{~ms}$ and the total experimental time is $200 \mathrm{~ms}$. Figure 5 illustrates both our broad spectral span — with bands centered at $386 \mathrm{~cm}^{-1}$ and at $1210 \mathrm{~cm}^{-1}$-and our resolution, which separates the neighboring transitions of toluene at $788 \mathrm{~cm}^{-1}$ and of cyclohexane at $802 \mathrm{~cm}^{-1}$.

Because the CARS signal scales with the cube of the peak power, the main difficulty associated with the use of laser systems of high-repetition frequency is related to the reduced pulse energy at the laser output. We compare the spectrum of toluene of Fig. 3(a) to the same spectrum (Fig. 6) measured with the setup described in [7], where the interferometric CARS signal was not heterodyned with the probe beam. It uses two 100$\mathrm{MHz}$ titanium-sapphire mode-locked lasers, which emit pulses of 20 -fs duration. In the spectrum of Fig. 6, the pulse energy at

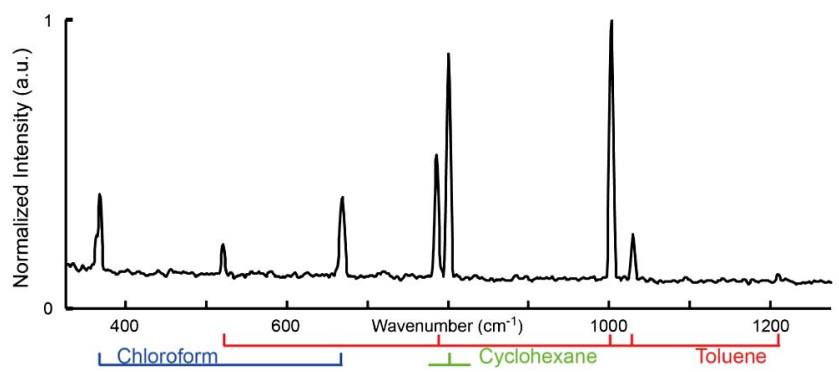

Fig. 5. Dual-comb CARS spectrum of toluene, chloroform and cyclohexane with a mixing ratio of $1: 1: 1$. 


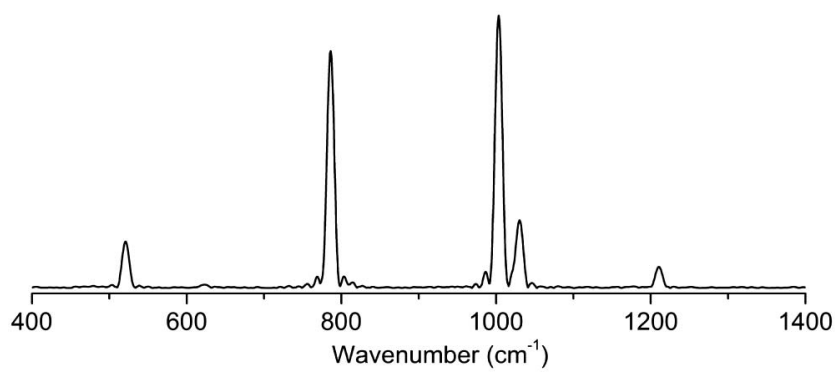

Fig. 6. Experimental dual-comb spectrum of toluene, measured with two 100-MHz femtosecond lasers.

the sample is $4 \mathrm{~nJ}$ per laser, the difference in repetition frequencies is $50 \mathrm{~Hz}$, and the apodized resolution is $6 \mathrm{~cm}^{-1}$. The recording time is $20 \mu$ s, which is identical to the spectrum shown in Fig. 3(a). The signal-to-noise ratio of the most intense toluene band at $1001 \mathrm{~cm}^{-1}$ is 1020 . Therefore, for the same measurement time, the spectrum recorded with the $1-\mathrm{GHz}$ dual-comb system [Fig. 3(a)] has a signal-to-noise ratio of about one order of magnitude worse than the spectrum measured with the $100-\mathrm{MHz}$ system whereas the pulse energy is eightfold weaker-therefore, the CARS signal is 512 times fainter. Such favorable scaling may be explained by several factors. The number of pump-probe sequences during the measurement time is 10 times higher with the $1-\mathrm{GHz}$ system than with that of $100 \mathrm{MHz}$. The heterodyne detection — by mixing with the local oscillator field of the spectrally overlapping probe laser beam-benefits the signal-to-noise ratio.

Such results contribute to a current effort to increase the acquisition speeds and rates of techniques of broadband nonlinear Raman spectroscopy (e.g., [23-28]). Moreover, direct frequency comb spectroscopy of samples in the condensed phase attracts growing interest [7-12]. Such experiments will benefit [29] from frequency combs of large line spacing and might trigger the development of dedicated laser sources. While linear-absorption dual-comb spectroscopy [9-11] may straightforwardly take advantage of such combs of highrepetition frequency, nonlinear dual-comb spectroscopy of transitions of short coherence times still calls for optimized strategies. In this Letter, we demonstrate that mode-locked lasers of $1-\mathrm{GHz}$ repetition frequency can be successfully harnessed for coherent Raman dual-comb spectroscopy of neat liquids. We experimentally show duty cycles that are 10-fold better than with lasers of a repetition frequency of $100 \mathrm{MHz}$ [7], as well as refresh times that are improved 20-fold. The span of the 1-GHz lasers of $1500 \mathrm{~cm}^{-1}$ allows, in principle, refresh rates $\delta f_{\text {rep }}$ up to about $10 \mathrm{kHz}$ but improvements to the signal-to-noise ratio will then be required. With such future enhancements to the signal-to-noise ratio and with the recent availability of commercial Ti:Sa femtosecond oscillators of $10-\mathrm{GHz}$ repetition frequency, the full potential of dual-comb coherent Raman spectroscopy without moving parts may be realized [30]. Broadband dual-comb Raman spectra measured on a microsecond-scale at a refresh rate of several tens of $\mathrm{kHz}$ may become within reach and dual-comb CARS might evolve into a powerful tool for studies of short-lived transient species, high-sensitivity experiments requiring efficient signal averaging, or fast hyper-spectral imaging.
Funding. European Research Council (ERC) (267854); Max Planck Foundation; Munich-Centre for Advanced Photonics (MAP).

Acknowledgment. We thank Dr. S. Holzner and A. Hipke for fruitful discussions and experimental advice.

\section{REFERENCES}

1. M. J. Thorpe, K. D. Moll, R. J. Jones, B. Safdi, and J. Ye, Science 311, 1595 (2006)

2. S. A. Diddams, L. Hollberg, and V. Mbele, Nature 445, 627 (2007).

3. I. Coddington, W. C. Swann, and N. R. Newbury, Phys. Rev. Lett. 100, 013902 (2008).

4. J. Mandon, G. Guelachvili, and N. Picqué, Nat. Photonics 3, 99 (2009).

5. B. Bernhardt, A. Ozawa, P. Jacquet, M. Jacquey, Y. Kobayashi, T. Udem, R. Holzwarth, G. Guelachvili, T. W. Hänsch, and N. Picqué, Nat. Photonics 4, 55 (2010).

6. A. Hipke, S. A. Meek, T. Ideguchi, T. W. Hänsch, and N. Picqué, Phys. Rev. A 90, 011805(R) (2014).

7. T. Ideguchi, S. Holzner, B. Bernhardt, G. Guelachvili, N. Picqué, and T. W. Hänsch, Nature 502, 355 (2013).

8. T. Ideguchi, B. Bernhardt, G. Guelachvili, T. W. Hänsch, and N. Picqué, Opt. Lett. 37, 4498 (2012).

9. T. Ganz, M. Brehm, H. G. von Ribbeck, D. W. van der Weide, and F. Keilmann, New J. Phys. 10, 123007 (2008).

10. A. Asahara, A. Nishiyama, S. Yoshida, K. Kondo, Y. Nakajima, and K. Minoshima, Opt. Lett. 41, 4971 (2016).

11. A. Ishizawa, T. Nishikawa, M. Yan, G. Millot, H. Gotoh, T. W. Hänsch, and N. Picqué, CLEO: 2015, OSA Technical Digest (online) (Optical Society of America, 2015), paper SW1G.7.

12. S. Avino, A. Giorgini, M. Salza, M. Fabian, G. Gagliardi, and P. De Natale, Appl. Phys. Lett. 102, 201116 (2013).

13. Y. Yan, E. B. Gamble, and K. A. Nelson, J. Chem. Phys. 83, 5391 (1985).

14. N. Dudovich, D. Oron, and Y. Silberberg, J. Chem. Phys. 118, 9208 (2003).

15. J. P. Ogilvie, E. Beaurepaire, A. Alexandrou, and M. Joffre, Opt. Lett. 31, 480 (2006).

16. B. von Vacano and M. Motzkus, Phys. Chem. Chem. Phys. 10, 681 (2008).

17. K. Isobe, A. Suda, M. Tanaka, H. Hashimoto, F. Kannari, H. Kawano, H. Mizuno, A. Miyawaki, and K. Midorikawa, Opt. Express 17, 11259 (2009).

18. T. H. S. Bican, H. W. Schrotter, and V. Mohacek Grosev, J. Raman Spectrosc. 26, 787 (1995).

19. M. Cui, J. Skodack, and J. P. Ogilvie, Appl. Opt. 47, 5790 (2008).

20. E. O. Potma, C. L. Evans, and X. S. Xie, Opt. Lett. 31, 241 (2006).

21. K. B. Wiberg and A. Shrake, Spectrochim. Acta A 27, 1139 (1971).

22. K. Kim and W. T. King, J. Chem. Phys. 80, 978 (1984).

23. C. H. Camp, Jr. and M. T. Cicerone, Nat. Photonics 9, 295 (2015).

24. S. R. Domingue, D. G. Winters, and R. A. Bartels, Opt. Lett. 39, 4124 (2014).

25. M. Tamamitsu, Y. Sakaki, T. Nakamura, G. K. Podagatlapalli, T. Ideguchi, and K. Goda, Vib. Spectrosc. in press (2016).

26. L. Czerwinski, J. Nixdorf, G. Di Florio, and P. Gilch, Opt. Lett. 41, 3021 (2016).

27. F. Saltarelli, V. Kumar, D. Viola, F. Crisafi, F. Preda, G. Cerullo, and D. Polli, Opt. Express 24, 21264 (2016).

28. J. Réhault, F. Crisafi, V. Kumar, G. Ciardi, M. Marangoni, G. Cerullo, and D. Polli, Opt. Express 23, 25235 (2015).

29. M. Yu, Y. Okawachi, A. G. Griffith, N. Picqué, M. Lipson, and A. L. Gaeta, "Silicon-chip-based mid-infrared dual-comb spectroscopy," preprint at arXiv:1610.01121 (2016).

30. G. Mélen, M. Yan, P.-L. Luo, T. W. Hänsch, and N. Picqué, Light, Energy and the Environment Congress, OSA Technical Digest (online) (Optical Society of America, 2016), paper FW2E.3. 